\title{
Transition from silicon nanowires to isolated quantum dots: Optical and structural evolution
}

\author{
Benjamin Bruhn,,${ }^{1, *}$ Jan Valenta, ${ }^{2}$ Ilya Sychugov, ${ }^{1}$ Kazutaka Mitsuishi, ${ }^{3}$ and Jan Linnros ${ }^{1}$ \\ ${ }^{1}$ Materials Physics, School of ICT, KTH Royal Institute of Technology, SE-164 40 Kista, Sweden \\ ${ }^{2}$ Faculty of Mathematics and Physics, Department of Chemical Physics and Optics, Charles University, \\ Ke Karlovu 3, 12116 Prague 2, Czech Republic \\ ${ }^{3}$ High Voltage Electron Microscopy Station, National Institute for Materials Science, Sakura 3-13, Tsukuba 3050003, Japan
}

(Received 26 September 2012; published 4 January 2013)

\begin{abstract}
The evolution of the structural and optical properties of a silicon core in oxidized nanowalls is investigated as a function of oxidation time. The same individual nanostructures are characterized after every oxidation step in a scanning electron microscope and by low-temperature photoluminescence, while a representative sample is also imaged in a transmission electron microscope. Analysis of a large number of recorded single-dot spectra and micrographs allows to identify delocalized and localized exciton emission from a nanowire as well as confined exciton emission of a nanocrystal. It is shown how structural transitions from one- to zero-dimensional confinement affect single-nanostructure optical fingerprints.
\end{abstract}

DOI: 10.1103/PhysRevB.87.045404

PACS number(s): 78.67.Bf, 81.16.-c, 62.23.Hj

\section{INTRODUCTION}

With the first discovery of efficient visible light emission from porous silicon, ${ }^{1}$ great interest in optical properties of silicon nanostructures arose and research in this field increased exponentially. Quantum confinement was identified as the cause for the efficient emission early on, ${ }^{1,2}$ and many other novel properties were discovered. However, despite intensive research for about two decades, the optical properties of silicon nanostructures are still not well understood. Using singledot spectroscopy, new phenomena like blinking, spectral diffusion, and atomic-like emission of individual emitters can be unveiled: properties that are usually obscured by averaging effects in ensemble measurements. Although time-consuming and difficult to perform, measurements on individual objects are required to enable detailed modeling and a deep understanding of the underlying mechanisms of light emission. At the same time, a sufficient number of single emitters must be analyzed in order to obtain statistical significance. If different properties are to be investigated in terms of correlation and causality, different measurements need to be performed on the same individual nanostructure.

In general, both silicon nanocrystals and nanowires are of interest for a range of applications such as photovoltaics, ${ }^{3}$ thermoelectrics, ${ }^{4,5}$ memory storage,${ }^{6}$ and medical applications like cancer cell imaging. ${ }^{7}$ In our previous work, ${ }^{8-11}$ nanostructures that allow for different and repeatable single-object characterization have been developed. It was shown that both oxidized silicon nanopillars and nanowalls can host luminescent nanocrystals and nanowires, spaced sufficiently for far-field optical measurements. In particular, it was found for silicon nanowalls that after a short oxidation they contain not only emitters of delocalized one-dimensional (1D) character, but also such that emit localized zero-dimensional (0D) photoluminescence (PL). ${ }^{12}$ This was ascribed to the coexistence of silicon nanowires and nanocrystals in the oxide wall, while the exact relation between the two remained unclear.

In this work we tracked and analyzed the same individual nanostructures throughout a series of oxidation steps, excluding sample-to-sample variations. Low-temperature PL and scanning electron microscopy (SEM) measurements, carried out after six subsequent oxidation steps, provide insight into the evolution of nanostructures in oxidized silicon wall systems. Transmission electron microscopy (TEM) imaging of a similar wall structure, representing different oxidation stages, sheds light on the transformation of nanowires and nanocrystals during this process, visualizing stand-alone nanocrystals, nanowires, and their hybrid systems in oxidized silicon walls. Through comparison of these data with recorded luminescence line shapes, a qualitative model describing the evolution and correlation of the structural and optical properties of luminescing silicon nanostructures was developed.

\section{EXPERIMENTAL DETAILS}

Sample preparation consisted of three main steps, namely, electron beam lithography, reactive ion etching, and oxidation in the self-limiting regime. First, a plain $10 \times 10 \mathrm{~mm} \mathrm{Si(110)}$ wafer piece ( $20 \Omega \mathrm{cm}$ resistivity) was cleaned with a short oxygen plasma etching step, followed by 6000-rpm negative resist (HSQ) spinning and $10-$ min baking at $150^{\circ} \mathrm{C}$. A pattern containing arrays of straight lines (sample A) was exposed on one sample; one with lines of undulating thickness on another (sample B). After exposure, the resist was developed in MF-CD26 for $1 \mathrm{~min}$. Both samples were etched by reactive ion etching in order to obtain silicon walls of approximately $250 \mathrm{~nm}$ height. The wall thickness approximates $70 \mathrm{~nm}$ in the case of straight walls and 50, 70, and $100 \mathrm{~nm}$ in the thin, medium, and thick compartments of the undulating walls, respectively.

Sample B was then oxidized for $3 \mathrm{~h}$ at $900^{\circ} \mathrm{C}$. Subsequently, it was sputtered with a carbon protection layer, and a wall with compartments of different thicknesses was removed from the sample with a focused ion beam and investigated in a transmission electron microscope. Sample A was initially oxidized for $30 \mathrm{~min}$ and then further oxidized in five steps of $15 \mathrm{~min}$, all at $900^{\circ} \mathrm{C}$. After each oxidation the sample was characterized using a PL setup and a scanning electron microscope. A few results after the initial oxidation step can be found in Ref. 12 . 
PL measurements were performed with a system comprised of an Olympus IX-71 inverted microscope with a $40 \times / 0.6$ objective lens (corrected for a thick cryostat window), an Acton SpectraPro 2358i imaging spectrometer, and a liquidnitrogen-cooled Princeton Instruments Spec-10:400B CCD camera. The lateral resolution of this system was limited by optical diffraction, i.e., $\sim 0.5 \mu \mathrm{m}$. For low-temperature spectral measurements the sample was placed in a Janis ST-500 cryostat. The sample was excited by an Omicron LDM405.120.CWA.L 405-nm cw laser diode at an excitation power density of $2.5 \mathrm{~W} \mathrm{~cm} \mathrm{~cm}^{-2}$. SEM measurements were performed with a Zeiss Gemini at $20-\mathrm{kV}$ acceleration voltage, using an in-lens detector, and TEM measurements with a JEOL 3000F field-emission transmission electron microscope operated at $300 \mathrm{kV}$.

\section{RESULTS}

As described in Sec. II, arrays of straight walls were oxidized in several steps and characterized by SEM and PL measurements after each iteration. Both these structures and an array of undulating walls that were used for TEM measurements are shown in Fig. 1. Naturally, even a straight silicon wall has some small thickness variations [see Fig. 1(d)], which are being amplified when the structure is oxidized at $900^{\circ} \mathrm{C}$. This temperature is in a range called the self-limiting regime, below the flow temperature of silicon dioxide. Upon oxidation, stress builds up, increasing or decreasing the oxidation rate at concave or convex surfaces, respectively. ${ }^{13-15}$ This effect leads to a nonuniform remaining silicon core within the silicon wall, as can be seen in the SEM images of an oxidized straight silicon wall in Fig. 2. The effect of self-limiting oxidation on silicon walls has also been mentioned in previous work. ${ }^{10,12}$

Figure 2 shows three sets of measurements, each set consisting of a PL image of a part of an oxidized silicon wall and a corresponding SEM image of the same structure. The same wall part is shown in all three sets and the oxidation

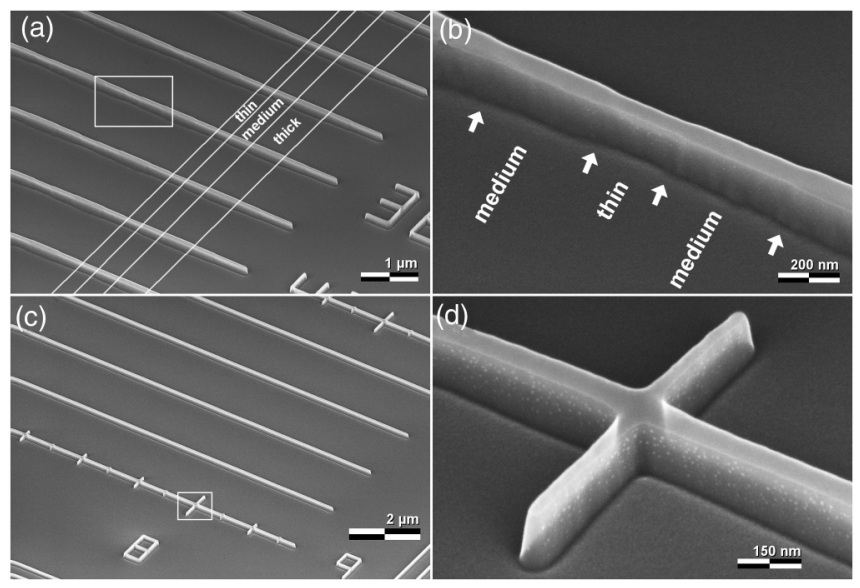

FIG. 1. SEM images of yet unoxidized silicon structures. (a) Array of walls with compartments of different thicknesses. (b) Closeup of the region indicated by the white rectangle in (a). One period of compartments is shown. (c) Array of straight walls where every fifth line contains orientation marks. (d) Closeup of the region indicated by the white rectangle in (c). Initial natural thickness variations can be seen along the walls.

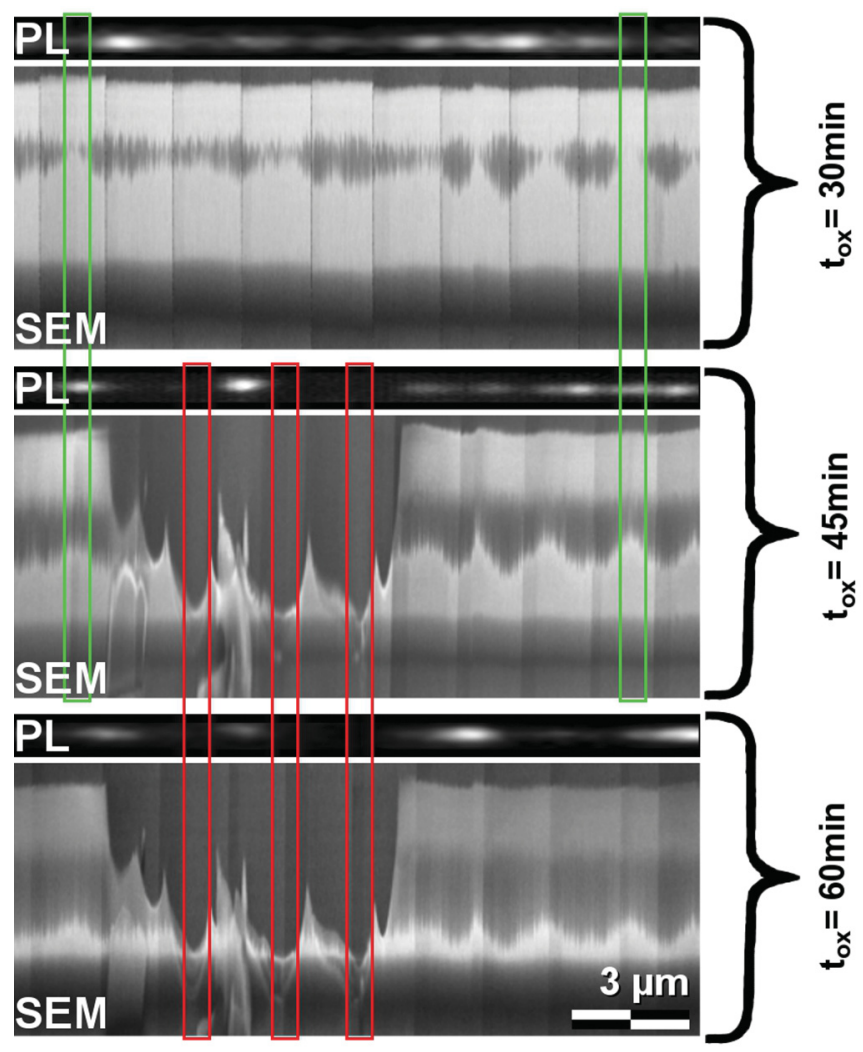

FIG. 2. (Color online) PL images and $50^{\circ}$ tilted view SEM micrographs for three different oxidation times of the same straight silicon wall. Silicon appears bright, whereas oxide looks darker and slightly transparent. In most oxidized regions PL emission can be observed. The far left and far right (green) rectangles indicate examples of nonoxidized parts of the wall with corresponding PL minima that turn into oxidized parts with corresponding PL maxima upon oxidation. The inner three (red) rectangles indicate broken parts of the wall, which correspond to missing PL emission. Note that the top part of the wall between the two left red rectangles lies next to the wall, which causes the PL signal from the luminescing nanostructures contained in it to appear slightly displaced from the wall axis (see PL image). Note that the SEM images are compressed horizontally and stretched vertically and that the scale bar thus only indicates the correct scale for the horizontal direction (the wall height is $\sim 250 \mathrm{~nm}$ ).

time is indicated on the right-hand side. From this figure it is possible to make the following observations. First, PL emission is not observed in spots where the wall is not oxidized through in the center, i.e., where the top and bottom part of the remaining silicon core are still connected. As soon as those parts disconnect upon further oxidation, though, strong PL emission can be detected, as indicated by the far left and far right (green) rectangles. Second, PL emission can be seen in many (arbitrary) positions within the oxidized parts of the wall. Both the top and the bottom part may play a role in this, especially where nanosized protrusions of the silicon core towards the wall center occur. Such protrusions can act as localization sites for excitons, therefore effectively becoming OD-like emission centers within a larger structure. Finally, the inner three (red) rectangles in Fig. 2 indicate destroyed parts of the wall, where no visible PL exists, confirming its origin from the nanowall interior. Note that previous work ${ }^{11}$ has shown that 
both insufficient and complete oxidation yield nonluminescing walls, further strengthening the conclusion that all PL emission stems from the silicon nanostructures contained in the oxidized silicon walls.

After a short oxidation, alongside localized bright emission spots, a broad spectral peak can be found all along the wall. This PL component can therefore be referred to as a delocalized spectrum and it is attributed to visible light emission from the undulating nanowire in the wall top. ${ }^{12}$ That the broad peak's intensity is comparable to that of the superimposed narrower spectrum and that the same spectral shape can be found at all points along the wire suggests that it does not originate from a local ensemble of quantum dots. Scattering
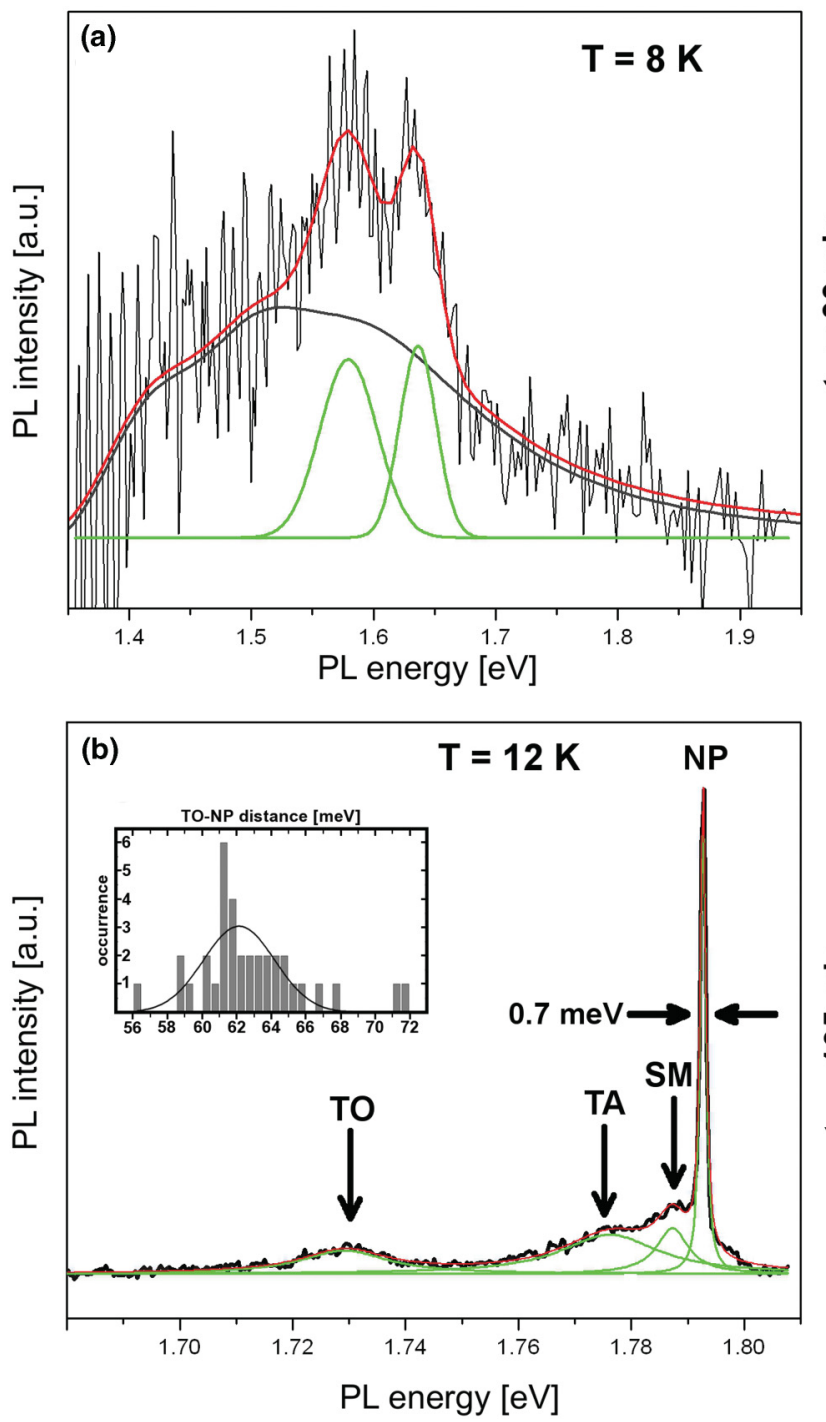

FIG. 3. (Color online) (a) Representative spectrum after a short oxidation at a low temperature. A broad delocalized component (dark gray) is prevalent in all spectra along the wall. Here, it is observed along with a localized emission peak and its TO replica [bottom two (green) curves]. (b) Representative single-nanocrystal spectrum measured at a low temperature after a long oxidation. The no-phonon (NP) peak FWHM (0.7 meV, deconvoluted) is clearly below $k_{B} T$ $(1.1 \mathrm{meV})$. Stretching mode and TA and TO phonon replicas can be observed. Inset: Histogram of the distance between NP and TO in a number of spectra, where the average is centered around $62 \mathrm{meV}$. effects due to insufficient isolation from the environment might cause significant broadening of the PL peaks from localized emission sites. However, the existence of two or more closely spaced quantum emitters in the same optically resolved spot cannot be completely eliminated as possible explanation. After longer oxidation the delocalized emission completely disappears and only localized spectra can be observed. The emission energy of the main peak and replica shifts parallel to the silicon band gap with temperature, proving that the observed emission is silicon related. Figure 3 shows typical spectra from different oxidation stages. While the delocalized component is visible and the fine structure of the localized spectra can often not be resolved at an early oxidation stage, the solely 0D-like spectra in a late oxidation stage exhibit an intricate fine structure where several distinct components can be distinguished. These are labeled NP, SM, TA, and TO for no phonon, stretching mode, ${ }^{16}$ transverse acoustical phonon peak, and transverse optical phonon peak, respectively. The spacings of SM, TA, and TO from NP are about 5, 16, and $61 \mathrm{meV}$, respectively. TA and TO phonon replicas, as well as the zero-phonon transition, are frequently reported in the literature for silicon nanocrystals (see, e.g., Ref. 17 and references therein). The latter can even be observed in bulk material containing shallow donors, ${ }^{18}$ due to exciton localization at the defect sites. In nanocrystals it is mainly attributed to exciton localization caused by quantum confinement. ${ }^{17}$ Note that the full width at half-maximum (FWHM) of the NP peak $(0.7 \mathrm{meV}$, deconvoluted $)$ is smaller than the thermal broadening $\left(k_{B} T=\right.$ $1.1 \mathrm{meV}$ ) at the temperature of $12 \mathrm{~K}$. Similar observations have been made in other works ${ }^{9,19}$ and can be regarded as strong evidence for single-nanocrystal emission, revealing its discrete nature of energy states. ${ }^{20}$ Further support for this claim is provided by previous ${ }^{21}$ and unpublished work that reports two-level blinking of many luminescing spots on similar samples, characteristic for single quantum emitters. The TO-phonon position does not exactly match the 56-meV

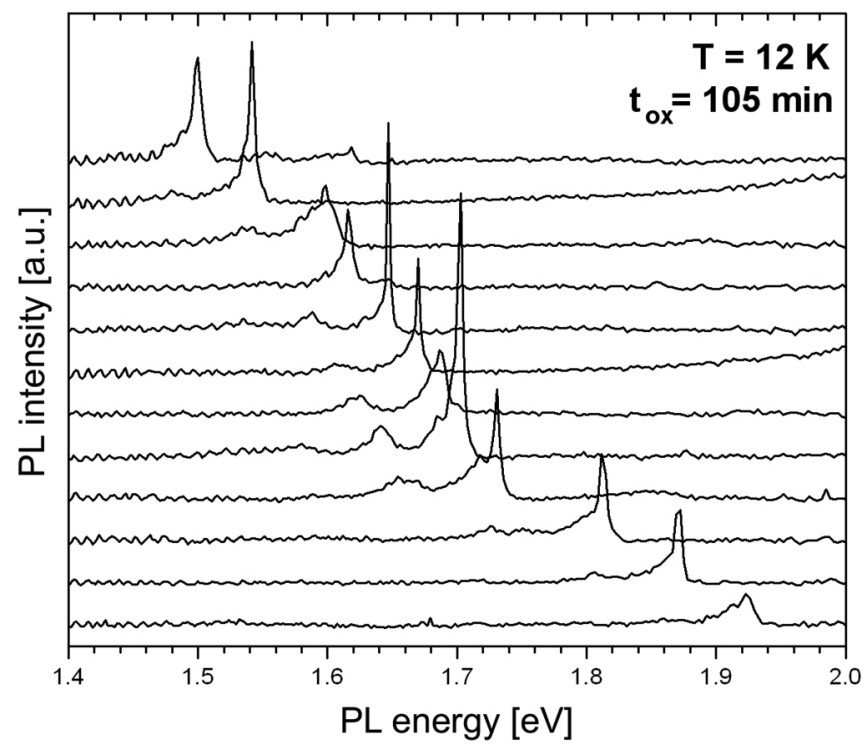

FIG. 4. Typical spectra measured on different nanowalls after 105 min of oxidation, covering the whole investigated emission energy range. Some of the peaks have an FWHM below $k_{B} T$, and some exhibit TA and TO phonon replicas. 
X-point TO phonon of bulk silicon. Instead, the spacing between the NP peak and the TO-phonon replica for different spectra reveal a broad distribution, centered at $\sim 62 \mathrm{meV}$ (see inset in Fig. 3). This shows that the bulk silicon band diagram does not accurately describe nanocrystalline silicon systems. The TO-phonon value at the $\Gamma$-point is $64 \mathrm{meV}$, so the observed distribution covers a full range of phonons, suggesting the effect of zone mixing in nanocrystallites. Such a broad distribution of replica positions was also observed in silicon nanocrystals prepared by thin-film oxidation. ${ }^{22}$ Not only the broad distribution of TO-NP energy spacing for different nanocrystals, but also the breadth of TA and TO replicas in a single dot suggest an influence of zone mixing. Another possible explanation for the TO-peak case is the participation of LO phonons, ${ }^{23,24}$ which assume similar energy positions as their TO counterparts, even though they are usually much less prominent. Note that strain at the $\mathrm{Si} / \mathrm{SiO}_{2}$ interface, which plays an important role in self-limiting oxidation, can influence the phonon frequency as well (see, e.g., Ref. 25).

Spectra with a distinct structure (cf. Fig. 3) are typically present at the latest oxidation stages. They cover the whole range of emission energies, as shown in Fig. 4. Some of the spectra consist of only the NP line, sometimes homogeneously broadened by effects like spectral diffusion or scattering, ${ }^{26}$ while others exhibit some or all of the aforementioned sidebands. Sychugov et al. ${ }^{19}$ report a blend of different spectra, some containing and some missing, e.g., TO-phonon replicas, and explain the dominance of zero-phonon transitions in silicon nanocrystal spectra by spatial localization of excitons, in addition to the quantum confinement effect. The interpretation of single peaks as zero-phonon transitions, as opposed to phonon-assisted transitions, can be justified by their narrow FWHM.

At different oxidation stages, low-temperature spectra were measured on the same wall and analyzed in terms of spectral position and width. Histograms of the emission energy and FWHM of all the localized spectra are displayed in Fig. 5. There might be a slight average shift towards higher emission energies for longer oxidation, but this trend does not seem to be significant. The spectral width of the emission peaks, on the other hand, does show a clear trend: On average it decreases significantly with continuing oxidation.

\section{DISCUSSION}

The fact that the average emission energy does not shift significantly (see Fig. 5, left) suggests an ongoing creation and annihilation of luminescent nanocrystals within the silicon wall. It seems that at all times there are silicon nanocrystals of different sizes to be found or, to be more precise, that the silicon wall contains excitons with different degrees of localization and confinement in different positions. Note,
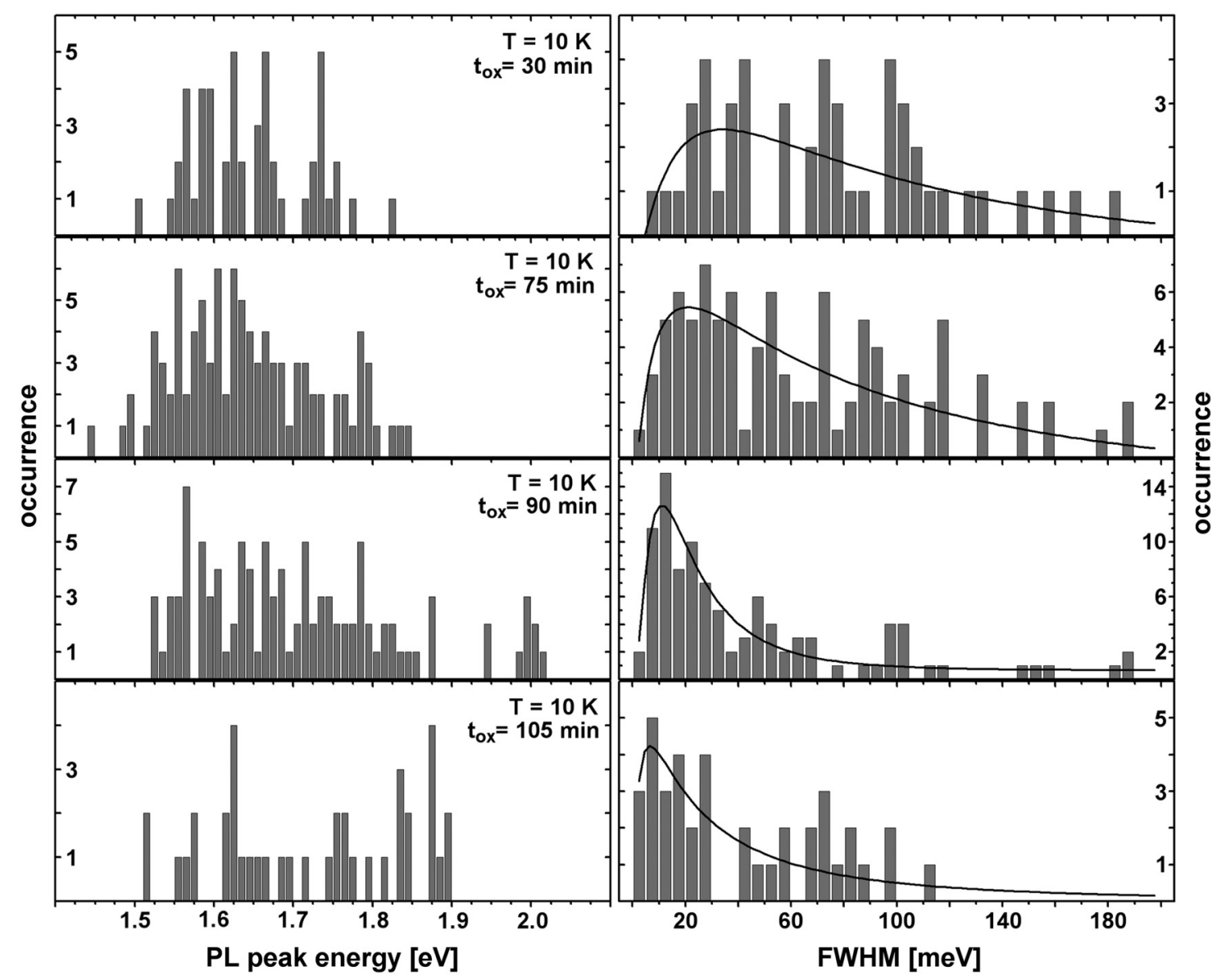

FIG. 5. Peak energy position and FWHM histograms for spectra measured on the same line after 30, 75, 90, and 105 min of oxidation. Log-normal functions were fit to the FWHM data as a guide for the eye, demonstrating a shift towards narrower spectra for longer oxidation. 
however, that it was not possible to follow an individual luminescing spot through several oxidation sequences, since oxidation durations of $15 \mathrm{~min}$ altered the local morphology so much that existing PL emitters were annihilated and others created. The substantial change in the average spectral width at different oxidation stages (see Fig. 5, right) suggests that the isolation of excitons from their surroundings becomes more efficient, so that less scattering and other interactions can occur. Mostly broad spectra in an early oxidation stage could be related to excitons localized at a curved interface or in a protrusion. Localized emission from excitons bound to impurities was observed a long time ago. ${ }^{18}$ It follows naturally that excitons bind to lattice defects at a rough and strained $\mathrm{Si} / \mathrm{SiO}_{2}$ interface. Interactions with phonons and other excitons in the nanowire can occur. On the other hand, narrow spectra in a late oxidation stage are likely to stem from separated silicon nanocrystals fully enclosed in a dioxide matrix, where the exciton cannot interact with other particles. Only after a long oxidation can many narrow spectra with an FWHM below the thermal broadening $\left(k_{B} T\right)$ be found.

In general, localized PL emission appears to be significantly brighter than the delocalized signal originating from nanowires. It is therefore reasonable to assume that localization increases the PL yield, possibly by decreasing the probability of nonradiative transitions in conjunction with an increased quasidirect transition. The latter would be caused by a spread of the electron and hole wave functions (and therefore a significant overlap) in $k$ space due to strong localization in real space. This $k$-conservation rule breaking has been

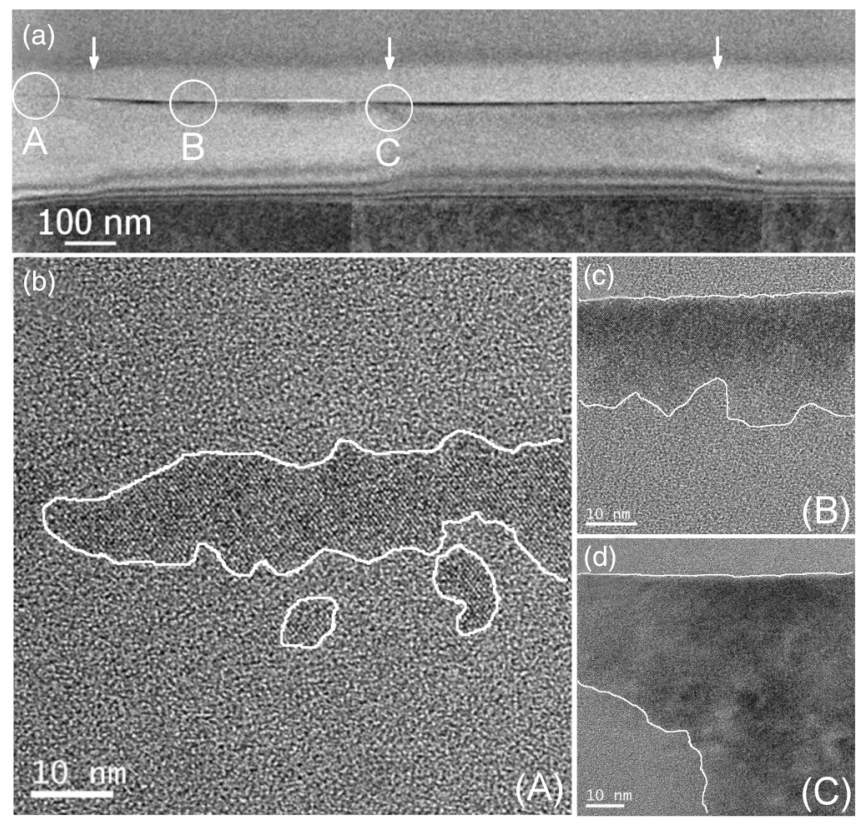

FIG. 6. Bright-field cross-sectional TEM micrographs of an oxidized silicon wall with compartments of three different thicknesses. Silicon appears as darker regions. (a) Overview, in which the white arrows mark boundaries between thin (left of left arrow), medium (right of right arrow, between left and center arrow), and thick (between center and right arrow) compartments. (b-d) Enlarged regions indicated by circles in (a). The remaining crystalline silicon lattice is outlined by white borders. calculated $^{24}$ and demonstrated ${ }^{27}$ for silicon nanocrystals and should generally apply for cases of confined and localized excitons.

In order to examine the geometry of the nanostructures within an oxidized silicon wall with a high resolution, TEM measurements were performed. Since the sample preparation is destructive, a sample other than the one used for the oxidation series experiment had to be used. In this case a wall with compartments of different thicknesses, as shown in Figs. 1(a) and 1(b), was used as a model system for different oxidation stages of a straight wall. Thicker parts oxidize more slowly, representing an earlier oxidation stage than more heavily oxidized thin parts. Figure 6(a) shows a piece of such an undulating wall with a thin compartment on the left side, followed by a medium, a thick, and another medium compartment. Figure 6(b) represents the transition to a late oxidation stage, where the nanowire in the top starts breaking apart, leaving a few well-separated nanocrystals behind. Figure 6(c) shows an intact nanowire with strong thickness variations and protrusions, which might act as localization centers for excitons and therefore pose quantum dots within the larger wire structure. Sivakovet al. ${ }^{28}$ also suggested this mechanism as being responsible for visible luminescence in silicon nanowires with a large diameter (and therefore insufficient quantum confinement).

TEM, SEM, and PL results together suggest that a silicon wall experiences several distinct stages upon oxidation. Initially, a nanowire with thickness variations is created in the wall top. This wire can contain bright localized visible light emission spots, alongside a broad spectrum that is distributed along the whole wire and most likely originates from delocalized 1D excitons. ${ }^{12}$ The localized emission is

(a)

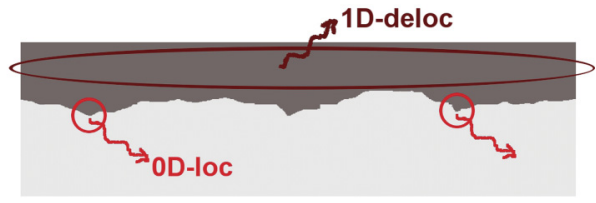

(b)

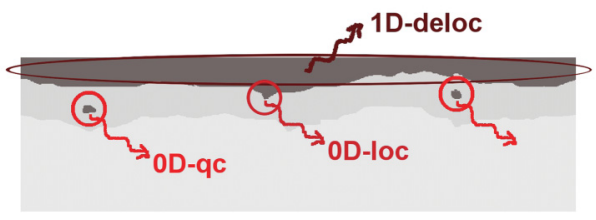

(c)

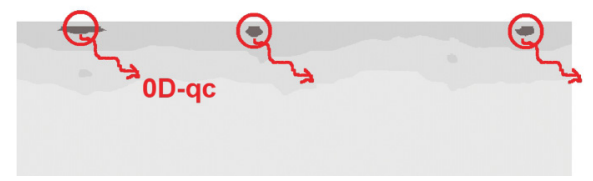

FIG. 7. (Color online) Schematic of the nanostructure evolution upon oxidation in the self-limiting regime. Bright parts represent oxide, whereas dark parts represent the remaining silicon core. Light emission is indicated by curved arrows. Three types of emission are shown, namely, delocalized 1D (1D-deloc) emission from nanowires, emission from excitons localized at a rough interface site (0D-loc), and emission from quantum-confined excitons that recombine within an isolated quantum dot (0D-qc). 
probably caused by exciton localization at defect sites at rough $\mathrm{Si} / \mathrm{SiO}_{2}$ interfaces. Then the wire starts to break up into smaller parts and some separated nanocrystals emerge, giving rise to emission from quantum-confined excitons. In the last luminescent stage the wire ceases to exist and only well-separated, isolated silicon nanocrystals are left. As has been shown by Sychugov et al. ${ }^{19}$ both quantum confinement and localization play a role in light emission from silicon nanocrystals. The observation of narrow spectra with a FWHM below the thermal energy $k_{B} T$ can be understood as a combination of the quantized nature of energy levels and weak interaction of excitons in well-isolated nanocrytals with other carriers. A schematic of the nanostructure evolution in a silicon wall upon oxidation, also indicating the different types of emission, is shown in Fig. 7.

\section{CONCLUSIONS}

Luminescent silicon nanostructures were created by electron beam lithography, reactive ion etching, and oxidation. A wall with compartments of different thicknesses was used for geometrical characterization using TEM. A straight silicon wall was oxidized in six subsequent steps, each of which was followed by PL and SEM measurements. At early oxidation stages, a broad spectrum along the whole wall could be found, which disappeared after sufficient oxidation. This spectral component can be attributed to light emission from the nanowire in the wall top. All oxidation steps yielded localized light emission spots, which can be attributed to localized excitons at lattice defect sites at the $\mathrm{Si} / \mathrm{SiO}_{2}$ interface in the nanowire (early oxidation stages) and confined and localized excitons in oxide-capped nanocrystals (later oxidation stages). The narrowing of the average localized emission peak FWHM upon oxidation, to be interpreted as reduced interaction of excitons with their surroundings, as well as TEM images showing wire morphology and wire/nanocrystal co-existence, support this picture. Spatially well-separated emission spots can be obtained throughout a large range of oxidation durations, which makes the oxidized silicon wall system exceptionally suitable for repeatable single-dot measurements. Note that these are crucial for gaining a deep understanding and enabling detailed modeling of the photophysics of oxide-capped silicon quantum dots. Nanocrystals in this system can be regarded as an especially suitable model system for porous silicon. Many properties, e.g., the typical PL line shapes and replicas and the zerophonon peak narrower than the thermal broadening at low temperatures, are similar to those of nanocrystals fabricated by other methods, which provides strong support for the existence of preparation-independent physics of oxidized silicon nanostructures.

\section{ACKNOWLEDGMENTS}

Financial support from the Swedish Research Council (V.R.) through an individual contract and through a Linné grant is gratefully acknowledged. Part of the work (J.V.) was supported by the EC project NASCEnT (GA No. 245977).
*Corresponding author: bbruhn@kth.se

${ }^{1}$ L. T. Canham, Appl. Phys. Lett. 57, 1046 (1990).

${ }^{2}$ V. Lehmann and U. Gösele, Appl. Phys. Lett. 58, 856 (1991).

${ }^{3}$ B. Tian, X. Zheng, T. J. Kempa, Y. Fang, N. Yu, G. Yu, J. Huang, and C. M. Lieber, Nature 449, 885 (2007).

${ }^{4}$ A. I. Boukai, Y. Bunimovich, J. Tahir-Kheli, J.-K. Yu, W. A. Goddard, and J. R. Heath, Nature 451, 168 (2008).

${ }^{5}$ A. I. Hochbaum, R. Chen, R. D. Delgado, W. Liang, E. C. Garnett, M. Najarian, A. Majumdar, and P. Yang, Nature 451, 163 (2008).

${ }^{6}$ Q. Li, X. Zhu, H. D. Xiong, S.-M. Koo, D. E. Ioannou, J. J. Kopanski, J. S. Suehle, and C. A. Richter, Nanotechnology 18, 235204 (2007).

${ }^{7}$ F. Erogbogbo, K.-T. Yong, I. Roy, G. X. Xu, P. N. Prasad, and M. T. Swihart, ACS Nano 2, 873 (2008).

${ }^{8}$ J. Valenta, R. Juhasz, and J. Linnros, Appl. Phys. Lett. 80, 1070 (2002).

${ }^{9}$ I. Sychugov, R. Juhasz, J. Valenta, and J. Linnros, Phys. Rev. Lett. 94, 087405 (2005).

${ }^{10}$ B. Bruhn, J. Valenta, and J. Linnros, Nanotechnology 20, 505301 (2011).

${ }^{11}$ B. Bruhn, F. Sangghaleh, and J. Linnros, Phys. Status Solidi A 208, 631 (2011).

${ }^{12}$ J. Valenta, B. Bruhn, and J. Linnros, Nano Lett. 11, 3003 (2011).

${ }^{13}$ R. B. Marcus and T. T. Sheng, J. Electrochem. Soc. 129, 1278 (1982).
${ }^{14}$ H. I. Liu, D. K. Biegelsen, F. A. Ponce, N. M. Johnson, and R. F. W. Pease, Appl. Phys. Lett. 64, 1383 (1994).

${ }^{15}$ H. Cui, C. X. Wang, and G. W. Yang, Nano Lett. 8, 2731 (2008).

${ }^{16}$ T. Takagahara, J. Lumin. 70, 129 (1996).

${ }^{17}$ D. Kovalev, H. Heckler, G. Polisski, and F. Koch, Phys. Status Solidi B 215, 871 (1999).

${ }^{18}$ P. J. Dean, J. R. Haynes, and W. F. Flood, Phys. Rev. 161, 711 (1967).

${ }^{19}$ I. Sychugov, J. Valenta, K. Mitsuishi, M. Fujii, and J. Linnros, Phys. Rev. B 84, 125326 (2011).

${ }^{20}$ S. V. Gaponenko, in Optical Properties of Semiconductor Nanocrystals (Cambridge University Press, Cambridge, 1998), pp. 111-117.

${ }^{21}$ B. Bruhn, J. Valenta, F. Sangghaleh, and J. Linnros, Nano Lett. 11, 5574 (2011).

${ }^{22}$ I. Sychugov, J. Valenta, K. Mitsuishi, and J. Linnros, Phys. Rev. B 86, 075311 (2012).

${ }^{23}$ O. J. Glembocki and F. H. Pollak, Phys. Rev. Lett. 48, 413 (1982).

${ }^{24}$ M. S. Hybertsen, Phys. Rev. Lett. 72, 1514 (1994).

${ }^{25}$ E. Anastassakis, A. Cantarero, and M. Cardona, Phys. Rev. B 41, 7529 (1990).

${ }^{26}$ S. A. Empedocles and M. G. Bawendi, J. Phys. Chem. B 103, 1826 (1999).

${ }^{27}$ D. Kovalev, H. Heckler, M. Ben-Chorin, G. Polisski, M. Schwartzkopff, and F. Koch, Phys. Rev. Lett. 81, 2803 (1998).

${ }^{28}$ V. A. Sivakov, F. Voigt, A. Berger, G. Bauer, and S. H. Christiansen, Phys. Rev. B 82, 125446 (2010). 